\title{
Cell wall biosynthesis impairment affects the budding lifespan of the Saccharomyces cerevisiae yeast
}

\author{
Mateusz Molon • Olga Woznicka · Jacek Zebrowski
}

Received: 16 September 2017/ Accepted: 28 November 2017/Published online: 30 November 2017

(C) The Author(s) 2017. This article is an open access publication

\begin{abstract}
The Saccharomyces cerevisiae yeast is one of the most widely used model in studies of cellular and organismal biology, including as aging and proliferation. Although several constraints of aging and budding lifespan have been identified, these processes have not yet been fully understood. Previous studies of aging in yeast have focused mostly on the molecular basics of the underlying mechanisms, while physical aspects, particularly those related to the cell wall, were rather neglected. In this paper, we examine for the first time, to our knowledge, the impact of cell wall biosynthesis disturbances on the lifespan in the budding yeast. We have used a set of cell wall mutants,

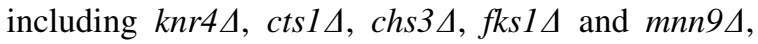
which affect biosynthesis of all major cell wall compounds. Our results indicated that impairment of chitin biosynthesis and cell wall protein mannosylation reduced the budding lifespan, while disruption in the 1,3- $\beta$-glucan synthase activity had no adverse
\end{abstract}

\footnotetext{
M. Molon ( $\bowtie)$

Department of Biochemistry and Cell Biology, University of Rzeszow, Zelwerowicza 4, 35-601 Rzeszow, Poland

e-mail: mateuszmolon@univ.rzeszow.pl

O. Woznicka

Department of Cell Biology and Imaging, Institute of

Zoology, Jagiellonian University, Krakow, Poland

J. Zebrowski

Department of Plant Physiology, Institute of

Biotechnology, University of Rzeszow, Rzeszow, Poland
}

effect on that parameter. The impact varied in the severity and the most notable effect was observed for the mnn $9 \Delta$ mutant. What was interesting, in the case of the dysfunction of the Knr4 protein playing the role of the transcriptional regulator of cell wall chitin and glucan synthesis, the lifespan increased significantly. We also report the phenotypic characteristics of cell wall-associated mutants as revealed by imaging of the cell wall using transmission electron microscopy, scanning electron microscopy and atomic force microscopy. In addition, our findings support the conviction that achievement of the state of hypertrophy may not be the only factor that determines the budding lifespan.

Keywords Yeast $\cdot$ Budding lifespan $\cdot$ Aging $\cdot$ Cell wall $\cdot$ AFM $\cdot$ SEM $\cdot$ TEM

\section{Introduction}

Saccharomyces cerevisiae is one of the most widely used model organism in the research of cellular processes, including aging and proliferation. Compared to mammalian cells, the budding fungi show specifically asymmetric cytokinesis, close mitosis and the presence of the cell wall, among which the latter may have considerable effect on aging (Lippuner et al. 2014; Steinkraus et al. 2008). It has been reported that some cell wall properties, including composition, size 
and surface wrinkling, may be age-associated (Cabib et al. 1997; Egilmez et al. 1990; Powell et al. 2000). Cell wall plays a multifunctional role in yeast's living processes (Gow et al. 2017; Lesage and Bussey 2006) and its synthesis, maintenance and remodelling is controlled by a large (above 1200) amount of genes (de Groot et al. 2001). The wall providing a relatively rigid envelope to the cell within the plasmalemma is essential for fungal cell growth, reproduction and interaction with environment. Particularly, it controls the cell's shape and growth rate, ensuring protection against external mechanical factors and internal osmotic pressure (Gow et al. 2017; Lesage and Bussey 2006). The wall polysaccharides provide the scaffold for surface glycoproteins which contribute to the adhesive wall properties and reduce wall permeability to large molecules, particularly wall digestive enzymes. Cell wall is a highly dynamic structure whose chemical composition and polymer interlinkage pattern respond to developmental changes and environmental cues. The Saccharomyces cerevisiae yeast cell wall is composed of external layer rich in $O$ and $N$-mannosylated proteins, imaged in the electron microscope as an electron dense part, and the layer adjacent to plasma membrane composed mostly of $\beta$ 1,3 glucans branched to some extent by $\beta-1,6$ bonds and a much smaller amount of chitin which interlinks the glucan polymers and other wall compounds into a load bearing-matrix (Klis et al. 2006; Smith et al. 2000).

A number of data indicate direct involvement of the cell wall in the reproductive processes, including budding (Cabib et al. 2001; Roncero and Sanchez 2010). Separation of mother and daughter cells in the budding yeast is essential for the organism's proliferation. This is preceded by formation of septa, a process closely linked to cytokinesis and division of nucleus. Following actomyosin ring contraction, a primary septum is formed which is built mainly of chitin fibres synthesised by chitin synthase II with Chs $2 p$ as a catalytic unit. Upon completion of this process, the secondary septum is laid down from both mother and daughter sides through the deposition of glucans and chitin as the major compounds. Synthesis of $\beta-1,3$ glucans is driven by glucan synthase Fks1 (Cabib et al. 2001; Lesage and Bussey 2006; Lesage et al. 2005) while the deposition of chitin requires chitin synthase Chs3 (Cabib et al. 2001; Cabib and Schmidt 2003; Ortiz and Novick 2006; Schmidt et al. 2002; Ziman et al. 1998). To enable physical separation of mother and daughter cells, both primary and secondary septa layers must undergo destruction and remodelling that is performed by digestive enzymes, including chitinase (Cts1) (Kuranda and Robbins 1991) and glucanases/glucosyltransferases. Formation and disassembly of septa employs, apart from some specific enzymatic complexes, a generally similar machinery that is used for synthesis and remodelling of the lateral cell wall. Thus, defects in the biosynthesis of particular cell wall compounds may affect septation and therefore the budding process in yeast cells. However, septation may take place even when either the primary septum is not formed or actomyosin ring contraction does not occur, and an alternative remedial septum, which is a form of the secondary septum, allows for performing separation of the mother and daughter cells (Cabib and Schmidt 2003; Tolliday et al. 2003). The budding process must undergo a coordinated control in respect of maintaining cell wall integrity, particularly in the region of the mother-bud neck. To meet this requirement and to prevent local cell surface extension, the chitin ring formed at the neck is bound to $\beta$-1,3-glucan in the cell wall (Arroyo and Arroyo 2013).

In 1959, Mortimer and Johnston were the first to discover that a single yeast cell ("mother") has limited reproductive potential (budding lifespan) (Mortimer and Johnston 1959). Earlier observation suggested that the new bud is never formed at the site of the older bud (Barton 1950). Therefore, the number of buds ("daughters") formed by a single cell defines the reproductive age. The first calculations predicted that yeast cannot produce more than 100 buds (Bartholomew and Mittwer 1953). The first estimations, technically still limited, allowed for observing 23 bud scars at the most (Barton 1950). Recent studies showed that in the case of some mutants, the "mother" cell can produce more than 70 buds (Molon et al. 2016). To this day, several factors have been identified as potential aging constraints (reviewed by Steinkraus et al. 2008), including accumulation of extrachromosomal rDNA circles (ERCs) (Sinclair and Guarente 1997), oxidative damage of protein (Aguilaniu et al. 2003) or thermal aggregates (Erjavec et al. 2007; Molon and Zadrag-Tecza 2016a). They were taken into account in various proposed hypotheses; however, none of them explains definitively the phenomenon of the reproductive potential limit. 
Cell wall involvement in longevity of fungi is still little understood. This may be putatively associated with the role of the wall in the control of volumetric growth and morphogenesis. One of the consequences of the choice of budding as the method of asexual reproduction is the continuous increase in cell volume. During subsequent doublings, the cell increases in size and changes its shape (Bartholomew and Mittwer 1953; Molon and Zadrag-Tecza 2016b; Powell et al. 2000; Zadrag-Tecza et al. 2009), which is to a great extent controlled by cell wall properties.

In this paper we present, for the first time to our knowledge, the effect of disturbances in cell wall biosynthesis on the budding lifespan of yeast. The obtained data emphasize the role of cell wall status, including possibly cell wall integrity, on the budding lifespan of yeast. We have explored mutations affecting all main cell wall compounds, including 1,3- $\beta$ glucans, chitin and mannoproteins. Additionally, we show morphological changes in the cell wall at the ultrastructural level revealed by scanning electron microscopy (SEM) and transmission electron microscopy (TEM) as well as atomic force microscopy (AFM) imaging. Taking into account the widespread contribution of cell wall characteristics to the growth, reproduction and sensing environment, linking the cell wall features to longevity may help better understand the complexity of the determinants of aging in $S$. cerevisiae.

\section{Materials and methods}

Yeast strains

The strains used in this study are given in Table 1 .
Growth conditions

Yeast cells were grown in a standard liquid YPD medium (1\% Difco Yeast Extract, 1\% Yeast BactoPeptone, $2 \%$ glucose) on a rotary shaker at $150 \mathrm{rpm}$, or on a solid YPD medium containing $2 \%$ agar. The experiments were carried out at the temperature of $28{ }^{\circ} \mathrm{C}$.

Determination of budding lifespan

Yeast budding lifespan was determined according to previously described procedure (Wawryn et al. 1999). Yeast cultures were grown in a rich YPD medium (1\% bacto-peptone, $1 \%$ yeast extract, $2 \%$ glucose, $2 \%$ agar) to the log phase. Five microliter aliquots of each culture was dropped on separate YPD plates. Forty single cells were micromanipulated for each experiment. Analysis were determined by micromanipulation using an optical microscope Nikon Eclipse E200 with attached micromanipulator. The number of buds formed by each cell was used to determine its reproductive potential. During the manipulation, the plates were kept at $28{ }^{\circ} \mathrm{C}$ for $16 \mathrm{~h}$ and at $4{ }^{\circ} \mathrm{C}$ during the night. The data represent the mean values from three separate experiments.

Scanning electron microscopy preparation

For scanning electron microscopy (SEM) the samples were fixed in $2.5 \%$ (v/v) glutaraldehyde GLU in $0.1 \mathrm{M}$ phosphate buffered saline (PBS) by $2 \mathrm{~h}$, rinsed with PBS $2 \times 10 \mathrm{~min}$ and dehydrated in graded alcohols. Finally, it was placed in transitional liquid (100\% acetone) and transferred to the Critical Point Drier (CPD E3000/E3100 Quorum Technologies). Then it was coated with gold using JFC-1100E Ion sputter (Jeol). For coating, the materials were placed on the holder with conductive carbon adhesive tabs (Electron
Table 1 Strains used in this study

\begin{tabular}{lll}
\hline Strain & Genotype & Source \\
\hline BY4741 & MATa his3 leu2 met15 ura3 & EUROSCARF \\
chs34 & MATa his3 leu2 met15 ura3 YBR023C $:$ kanMX4 & EUROSCARF \\
cts14 & MATa his3 leu2 met15 ura3 YBR023C : kanMX4 & EUROSCARF \\
fks14 & MATa his3 leu2 met15 ura3YLR342 W::kanMX4 & EUROSCARF \\
knr4A & MATa his3 leu2 met15 ura3 YGR229C::kanMX4 & EUROSCARF \\
mnn94 & MATa his3 leu2 met15 ura3 YPL050C::kanMX4 & EUROSCARF \\
\hline
\end{tabular}


Microscopy Sciences). Morphological characters were analyzed by means of Scanning Electron Microscope (JSM-5410).

Transmission electron microscopy preparation

Samples were fixed in a primary fixative $2 \%(\mathrm{v} / \mathrm{v})$ glutaraldehyde, $2.5 \%(\mathrm{v} / \mathrm{v})$ paraformaldehyde in a cacodylic buffer and were postfixed for $2 \mathrm{~h}$ in $2 \%$ $\mathrm{OsO}_{4}$. Next, samples were dehydrated in an alcohol series and twice in propylene oxide before being embedded in Poly/bed 812 resin (Polysciences). Embedded samples were sectioned with a Reichert Ultracut and then observed by using the transmission electron microscopy. For determination of yeast cell wall thickness images were captured with the microscope equipped with the TVIPS digital camera. Cell thickness was measured using the EM MENU4 software.

\section{Atomic force microscopy}

Saccharomyces cerevisiae cells were collected at the exponential growth stage, three times washed in PBS buffer and deposited on a microscopic cover glass. Subsequently, they were dried under $\mathrm{N}_{2}$ atmosphere at ambient temperature. AFM topographical imaging was performed in air in the PeakForce Tapping mode using the BioScope Catalyst II system with the Nanoscope V controller (Veeco Instruments, Santa Barbara, CA, US) and silicon nitride MLCT probes (Bruker, Camarillo, CA). The Height and PeakForce Error images were obtained at the scan rate of $0.33 \mathrm{~Hz}$ and with 512 pixels per line using the Nanoscope (1.40 v.5, Bruker) software and the ScanAsyst algorithm for the optimization of the gain and setpoint parameters. The images were processed using the Nanoscope Analysis v. 1.50 (Bruker Co.) software.

Estimation of cell volume

Cell volume was estimated by optical microscopy and analysis of images collected every fifth cell budding during the routine procedure of determining the reproductive potential. The images were captured with the Nikon Eclipse E200 microscope equipped with the Olympus DP26 digital camera. Cell diameter (d) was measured using the Olympus cellSens Standard software in various planes for each cell and the mean value was used for calculations. Assuming that each cell has a regular shape similar to the sphere, the cell volume $(\mathrm{V})$ was calculated as $\mathrm{V}=4 / 3 \pi(\mathrm{d} / 2)^{3}$.

Phenotypic analysis - a spot test for sensitivity to Congo red, Calcafluor White, MMS and sodium chloride

Yeast cultures were grown to exponential phase $\left(\mathrm{OD}_{600 \mathrm{~nm}}\right.$ between 0.8 and 1$)$ and serially diluted to different cellular concentrations as indicated. Five microliters of each cell suspension was spotted onto agar plates containing various concentrations of Congo red (Sigma-Aldrich), Calcafluor White (Sigma-Aldrich), methyl methanesulfonate (SigmaAldrich) and sodium chloride (Sigma-Aldrich). Growth was registered $48 \mathrm{~h}$ after incubation at $30{ }^{\circ} \mathrm{C}$. All phenotypes described in this work were confirmed by multiple tests.

Statistical analysis

The results represent the mean \pm SD values for all cells tested in two independent experiments ( 80 cells). The differences between the mutant strain compared to the wild-type strain were estimated using a one-way ANOVA and Dunnett's post hoc test. The values were considered significant if $p<0.01$. Statistical analysis was performed using the Statistica 10 software.

\section{Results}

The budding lifespan of the cell wall mutants

To obtain insight into the role of cell wall in aging of the $S$. cerevisiae yeast, we examined strains that were impaired in the process of cell wall synthesis. Initially, we analysed the budding lifespan measured as the number of daughter cells produced by the mother cell. As seen in Fig. 1, disturbances in the regulation of cell wall synthesis had a significant impact on the budding lifespan. Cell wall mutations considerably altered cell lifespan at the exponential growth phase, significantly

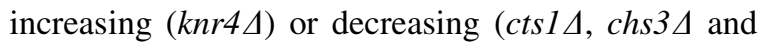
mnn94) the lifespan compared to the BY4741 strain (Fig. 1). Only the impairment in 1,3- $\beta$-glucan synthase activity $(f k s 1 \Delta)$ did not affect the budding lifespan. The most dramatic reduction of the trait 


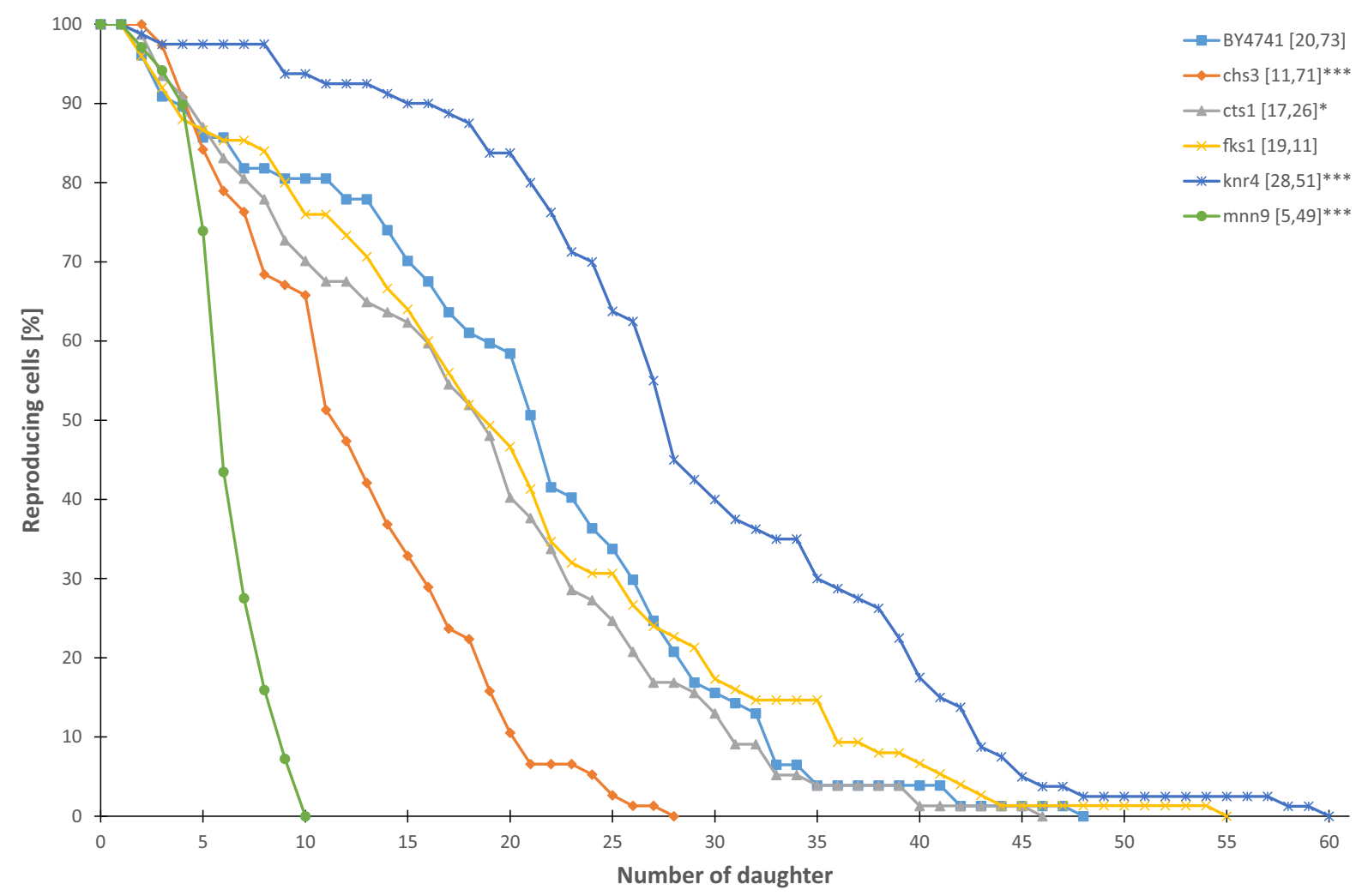

Fig. 1 Comparison of the reproductive potential of the haploid wild type yeast strain BY4741 and isogenic mutant strains chs34, cts1 1 , fks1 1 , knr4A, mnn94. Mean values (for total 80

(almost 4 times compared with BY4741) was observed for the mnn94 mutant defective in protein mannosylation. Moderate impact of the cell wall mutant on the budding lifespan was observed for knr4 4 , chs $3 \Delta$ and cts $1 \Delta$ strains. Interestingly, more than half the population of $\operatorname{chs} 3 \Delta$ exploded during a routine procedure determining the reproductive potential (data not shown). Thus, knock-out of the nonessential function of cell wall-related genes had an effect on the budding lifespan (Fig. 1).

Changes in the cell volume during budding lifespan

Next, we tested changes in cell volume during subsequent cycles of the mutant and wild-type strains. In view of the hotly debated influence of cell volume on the budding lifespan, we obtained data which may throw new light on the hypertrophy hypothesis. Our data show that the basic assumptions of the hypothesis cells from two independent experiments) of the reproductive potential are shown in parentheses

may not be obvious. As seen in Fig. 2, the $f k s l \Delta$ and knr4 $\Delta$ mutants achieved a similar maximum volume to that of the wild-type strain when they finished budding. However, in the case of chs $3 \Delta$, cts $1 \Delta$ and $m n n 94$, the maximum volume was about $50 \%$ lower compared to the wild-type strain. Consequently, for this group of strains, a higher cell volume was associated with a relatively higher budding lifespan.

Estimation of the cell wall thickness determined by TEM

Attempting to explain these changes in terms of cell wall properties, we analysed several morphological parameters of the wall, including thickness, roughness, and Young's modulus as well as cell volume. Cell wall thickness determined on the basis of the electron microscope measurements did not vary in $m n n 9 \Delta, k n r 4 \Delta$ and chs34 but increased in the cts $1 \Delta$ and $f k s 1 \Delta$ strains compared to wild-type cells (Fig. 3). 


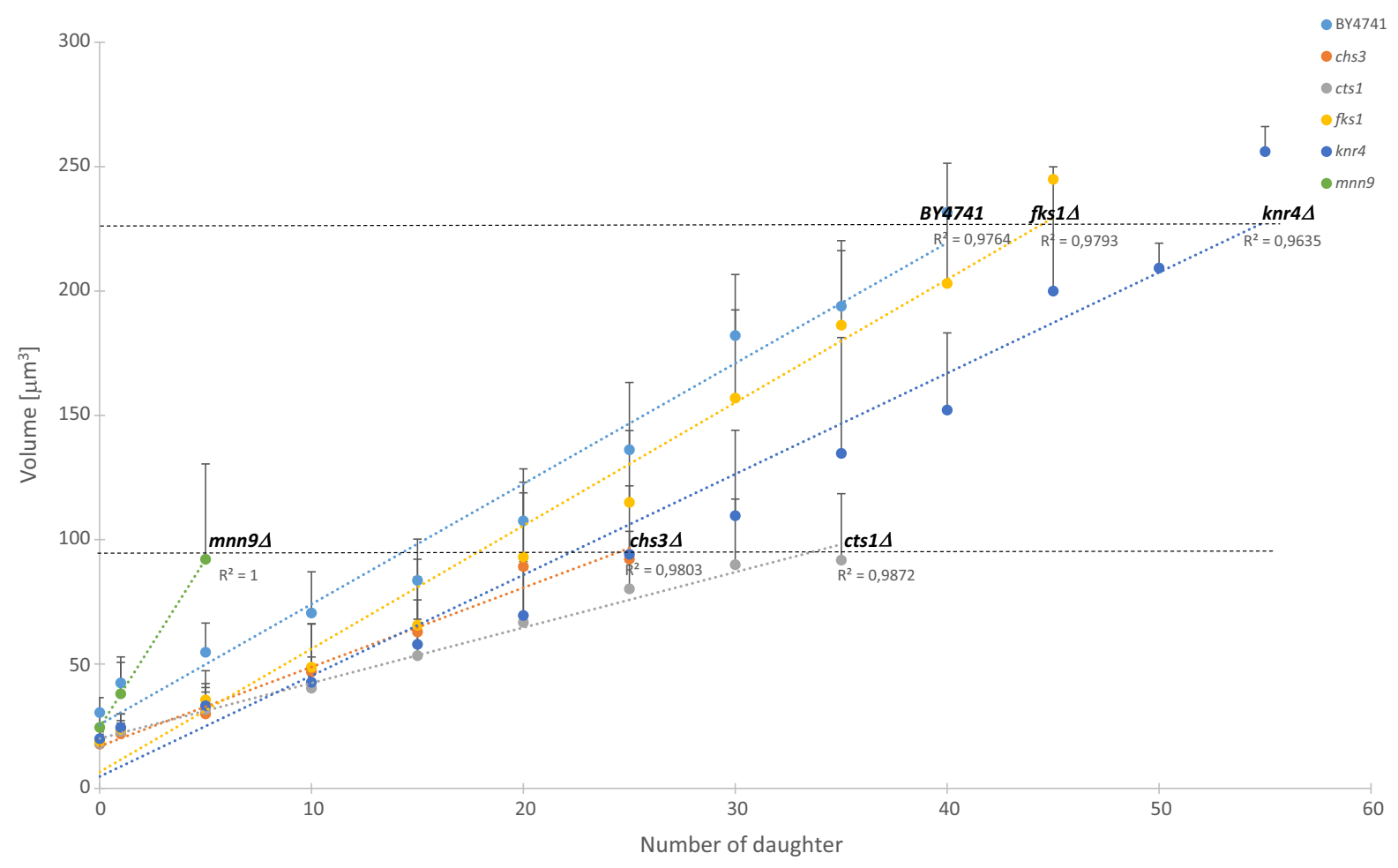

Fig. 2 Dependence of cell volume on the number of daughters accomplished by mother yeast cells. The results represent values for all cells tested in two independent experiments (80 cells). The bars indicate SD

Fig. 3 Comparison of cell wall thickness between BY4741 (wild-type) and cell wall mutants (chs34, cts $1 \Delta$,

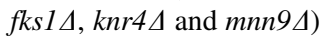
measured in the images from the transmission electron microscopy using the EM Menu4 software. The means were obtained for 15 cells. The bars indicate SD.

Statistical significances were assessed using ANOVA and the Dunnett's post hoc test $(* * \mathrm{p}<0.05$, $* * * \mathrm{p}<0.001)$

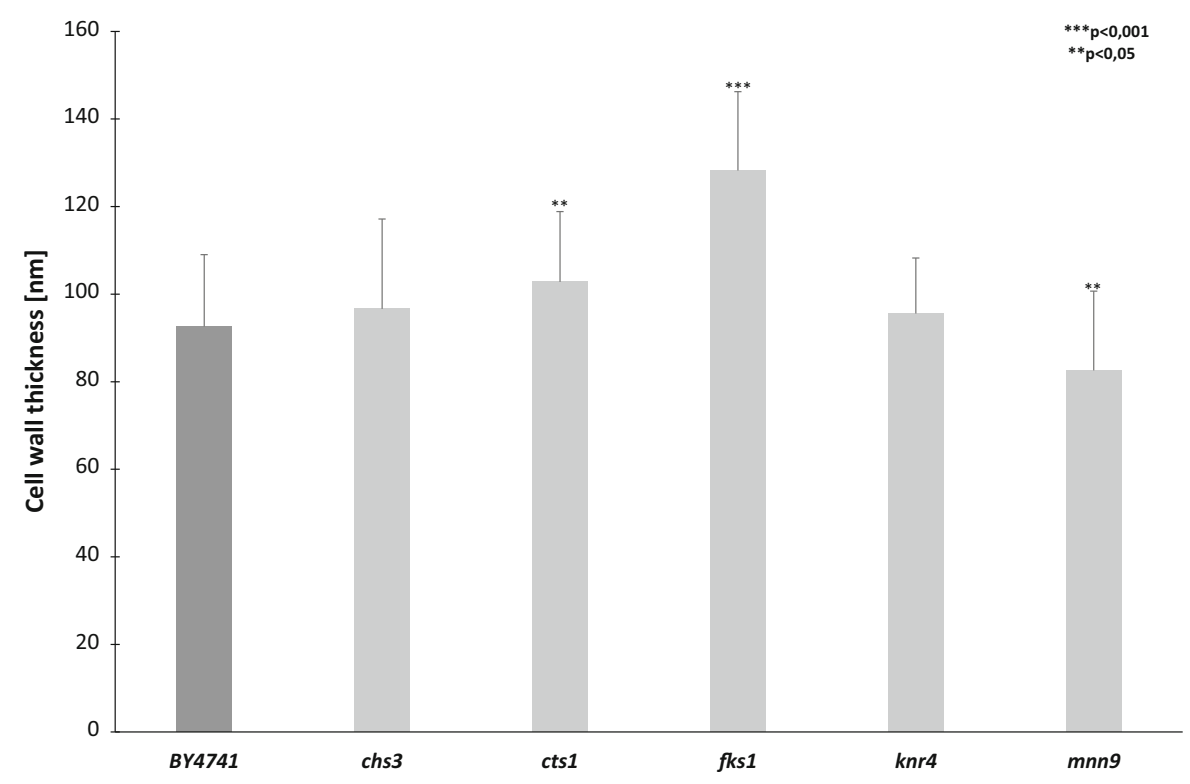

However, the lifespan parameter did not correlate with cell wall thickness. The mnn94 mutant displaying a dramatically reduced lifespan showed similar wall thickness as wild-type, while the highest increase in the cell wall thickness was observed in the $f k s 1 \Delta$ mutant in which the lifespan was not affected. 
Cell wall surface characterisation

For inspection of possible deformations in the cell wall surface, e.g. collapse, protrusions or breakage, we used scanning electron microscopy (SEM) and atomic force microscopy (AFM) as two complementary tools. SEM micrographs of wild type cells and cell wall mutants are given in Fig. 4. Cells of the mutants differed to some extent in shape, volume and bud scar morphology compared to the wild-type strain. The most striking differences in the wall topography were observed for $f k s 1 \Delta$ cells, which showed some protrusions in the form of wrinkles. We also noticed that the chs $3 \Delta$ and cts $1 \Delta$ mutants had abnormal bud scarring (Fig. 4b). The AFM imaging did not show clear modifications of cell wall topography in the examined mutants at the exponential phase of growth (Fig. 5). Representative images collected in the Height mode are given in the upper row, while corresponding images obtained in the Peak Force mode are provided

a
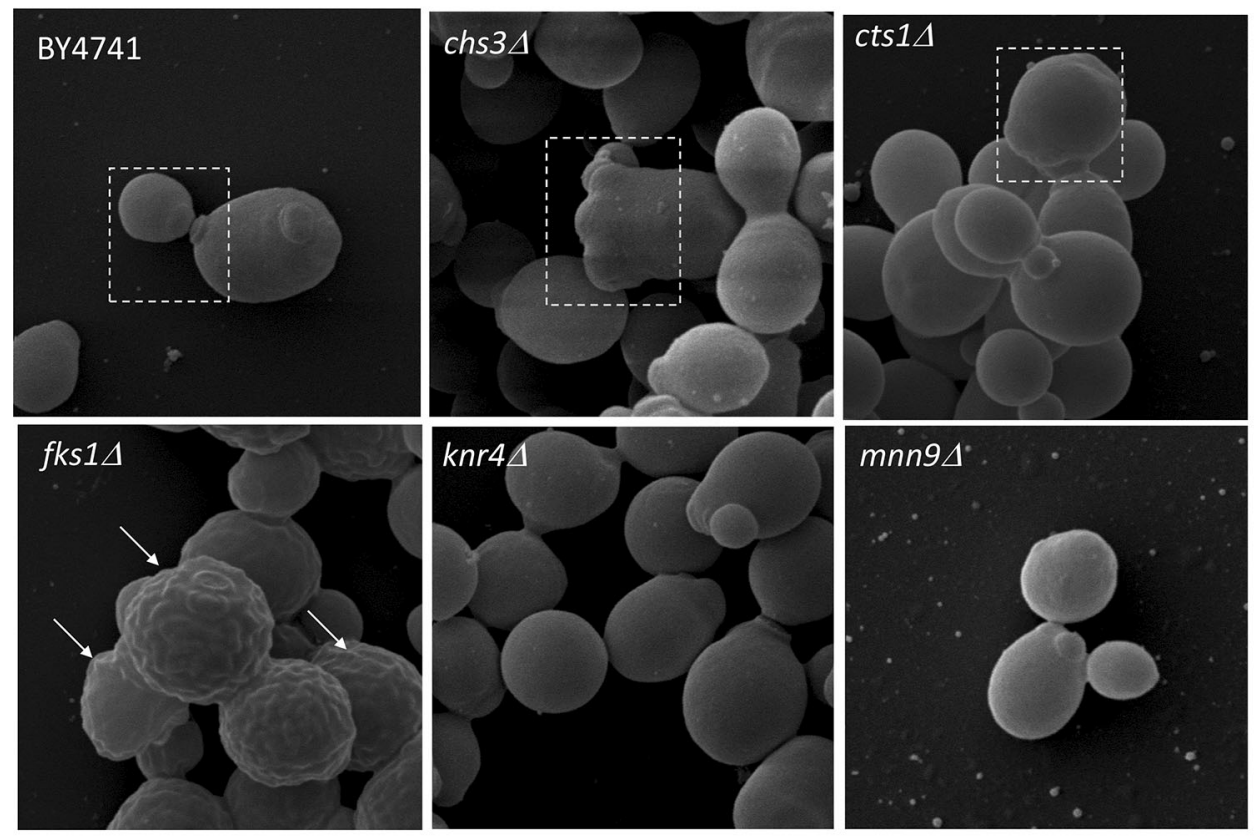

beneath. The latter mode is particularly sensitive to homogeneities in the wall material, especially those that are related to the mechanical surface properties. Interestingly, the presence of bulges and protuberances on the surface that could be seen in SEM micrographs for some $f k s l \Delta$ cells was not confirmed in the AFM imaging in either the Height or the Peak Force modes. This may suggest possibility of their incidental occurrence or formation resulting of specific sample preparation.

Further, we analysed a possible relationship between elastic properties of the cell wall and the lifespan parameter. Young's modulus (E) of cell wall obtained through nanoindentation was taken from the literature (Dague et al. 2010). This parameter was generally lower in the examined mutants compared to the BY4741 strain, with the exception of the mnn94 strain. Therefore, the most dramatic reduction of the reproductive potential observed for the $m n n 9 \Delta$ cells was not reflected in the changes of the modulus.

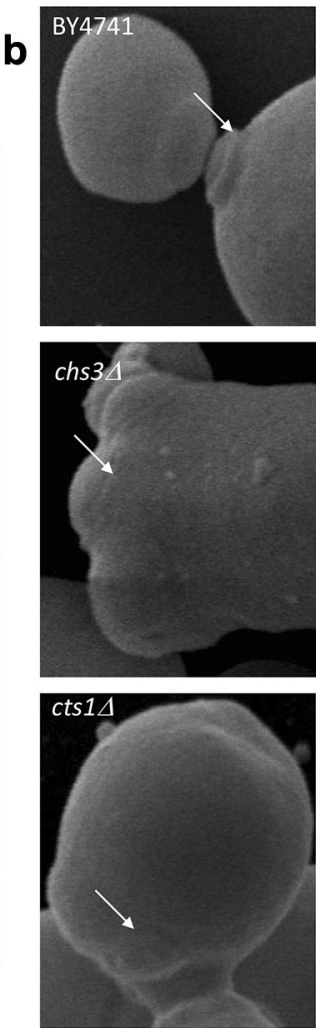

Fig. 4 Scanning electron microscopy images of Saccharomyces cerevisiae cells for the BY4741 wild-type strain and cell wall mutants: chs $3 \Delta$, cts $1 \Delta, f k s 1 \Delta, k n r 4 \Delta$, and mnn9A. The arrows in a indicate wrinkles on the cell wall surface observed in $f k s 1 \Delta$. The arrows in $\mathbf{b}$ indicate changes in bud scars structure occurring in the chs $3 \Delta$ and cts $1 \Delta$ mutants compared to wildtype 


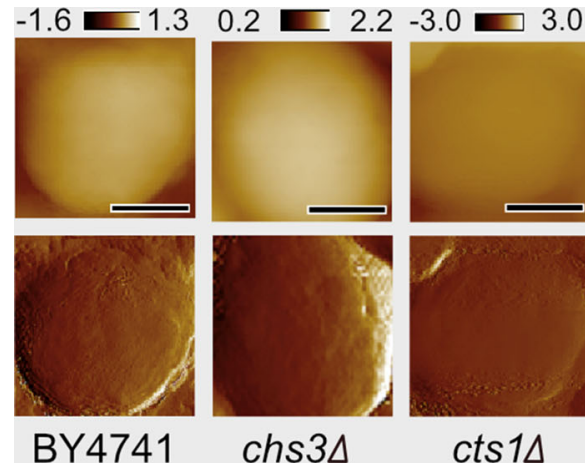

Fig. 5 Representative AFM images of Saccharomyces cerevisiae cell surface for the wild type strain BY4741 and five cell

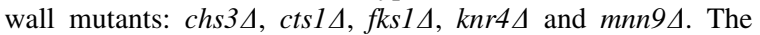
upper array of photos was collected in the Height mode. The

Moreover, reduction in E reported for the knr4 4 and $f k s 1 \Delta$ strains corresponded both to the increased and non-altered lifespans, respectively. Cell wall roughness reported in literature (Dague et al. 2010) was generally much higher in the mutants compared to BY4741, with the exception of $f k s 1 \Delta$. The $f k s 1 \Delta$ mutant showed a reproductive potential similar to that of the wild-type strain, while the mnn94 mutant, characterised by the highest increase in roughness, displayed the lowest reproductive potential among the strains. In turn, increase in roughness reported for the chs $3 \Delta$ and knr4 $\Delta$ strains corresponded to the reduced and extended reproductive potentials, respectively. Taken altogether, these data indicate lack of clear association between the reproductive potential and roughness and elastic properties of the wall surface.

Sensitivity to agents disturbing the wall biosynthesis

Finally, to examine whether the mutants truly had defects in cell wall biosynthesis, we conducted spotted tests on media containing Calcofluor white $(\mathrm{CW})$ or Congo red (CR). Both are known to disturb cell wall biosynthesis in normal strains. The fks $1 \Delta$ and knr4 $\Delta$ strains were hypersensitive to $\mathrm{CW}$ and $\mathrm{CR}$, and the $f k s 1 \Delta$ strain appeared to be slightly less sensitive than knr4A. Additionally, the chs34 mutant was hyperresistant to both $\mathrm{CW}$ and $\mathrm{CR}$. Moreover, the impairment of the analysed cell wall-related genes did not affect the growth of the cells under methylation agent (0.01\% Methyl methanesulfonate) and osmotic stress (0.5 $\mathrm{M}$ and $1 \mathrm{M} \mathrm{NaCl}$ ) (Fig. 6).

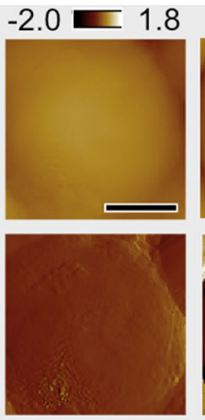

fks $1 \triangle$

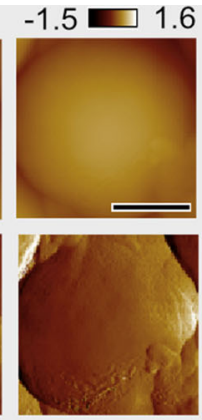

$k n r 4 \Delta$

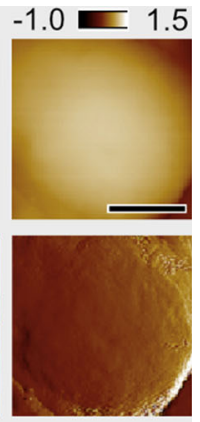

$m n n 9 \Delta$

height scale above the photos is given in $\mu \mathrm{m}$. The corresponding lower array of images was obtained in the Peak Force Error mode. The scale bar $=1 \mu \mathrm{m}$

\section{Discussion}

Involvement of the $S$. cerevisiae yeast cell wall in cell growth and reproduction, e.g. formation of septum and mother-daughter cells separation, is quite well understood (reviewed by Cabib et al. 2001; Roncero and Sanchez 2010) However, the relationship between composition, molecular organisation and physicochemical properties of the wall and cell longevity has not been studied extensively so far. To address that problem, we have examined the effect of several wall mutations, including chs $3 \Delta$, cts $1 \Delta, f k s 1 \Delta, k n r 4 \Delta$ and $m n n 9 \Delta$, on the budding lifespan of yeast cells. Both the deposition of primary and secondary septa as well as disassembly of the structures engages the machinery of cell wall synthesis and decomposition, which is typically employed into formation and remodelling of the lateral cell wall.

In earlier studies, which attempted to link aging to septation, the focus was given to bud scar characteristics. Bud scars provide a means of determining the reproductive age of cells (Barton 1950; Egilmez et al. 1990; Sinclair et al. 1997). Mortimer and Johnston (1959) suggest that accumulation of chitin (in bud scars) may limit budding. Later data disproved that theory and demonstrated that scarring is a result rather than cause of replicative aging (Egilmez and Jazwinski 1989). More recent data suggest that chitin scar breaks during aging, which may result from the bud scarring's reduced capacity of stretching despite chitin network elasticity (Powell et al. 2003).

Interestingly enough, our study showed that strains defective in genes involved in wall synthesis or 

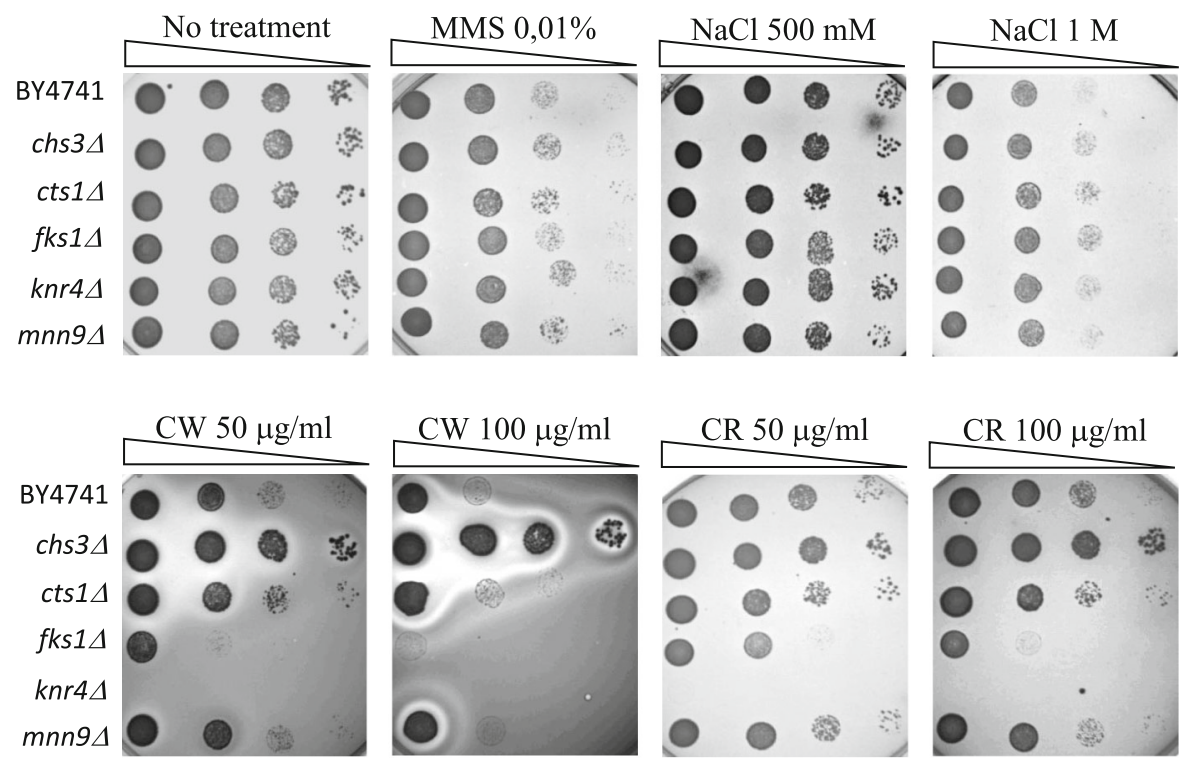

Fig. 6 Sensitivity of the BY4741 strain and cell wall mutants with disrupted $C H S 3, C T S 1, F K S 1, K N R 4, M N N 9$ genes to Calcofluor white (CW), Congo red (CR), Methyl methanesulfonate (MMS) and sodium chloride $(\mathrm{NaCl})$. Yeast strains were grown in YPD medium $\left(28^{\circ} \mathrm{C}\right)$, spotted onto YPD plates

remodelling may either increase or decrease the budding lifespan parameter to various degrees. Exceptionally, glucan synthesis impairment ( $f k s 1 \Delta$ ) had no effect on lifespan. The Fks1 protein is a catalytic subunit of the $\beta$-1,3-D-glucan synthase involved in polymerisation of the main structural polysaccharide, which may represent up to $80 \%$ of the dried wall weight, and takes part in wall synthesis, maintenance and remodelling (Utsugi et al. 2002). S. cerevisiae has three glucan synthase genes but Fks1p functions as the major $\beta$-1,3-glucan synthase during vegetative growth in yeast (Klis et al. 2006). It is, however, one of functionally alternative subunits of the $\beta$-1,3-D-glucan synthase complex. The other protein, Gsc2/Fks2, may repossess the main function in polymerisation of the glucan depending on the environmental conditions (Klis et al. 2002). Fks1p is directly involved in the budding process, being responsible for deposition of $\beta$-1,3-D-glucans during formation of the secondary septa. Although the mutation did not alter the lifespan and the final cell volume, the wall formed by the mother cells was thicker relative to the wild-type strain according to our TEM measurements, and of reduced stiffness and increased roughness at the stationary stage as reported by Dague et al. (2010). containing the indicated amounts of $\mathrm{CW}, \mathrm{CR}, \mathrm{MMS}, \mathrm{NaCl}$ and incubated at $28{ }^{\circ} \mathrm{C}$. Growth on YPD agar plates was treated as a control. Representative results from two independent experiments are shown

The majority of the examined mutants, including cts $1 \Delta$, chs $3 \Delta$, and mnn9A, decreased the budding lifespan. Moderate reduction in the lifespan was observed for chs $3 \Delta$ and $\operatorname{cts} 1 \Delta$ mutants, which were defective in chitin biosynthesis and chitinase activity, respectively. Chitin is a compound that represents only $1-2 \%$ of the dry weight of wall in normal growth but may show considerably enhanced levels in response to the altered environment or altered wall properties due to genetic disturbances (Carotti et al. 2002; Klis et al. 2006; Popolo et al. 1997). Despite low abundance of chitin, the interlinks established between glucans and also proteins provide the wall with appropriate strength and structural integrity (Klis et al. 2006; Orlean 2012). The CHS3 comprises a transmembrane catalytic unit responsible for a great majority of chitin synthesis during normal growth and is involved in the chitin stress response (Bulik et al. 2003). It also takes part in the deposition of the chitin-containing ring at the base of the emerging bud (Shaw et al. 1991). Moreover, Chs3p along with Fks1p is involved in the deposition of secondary septa on both daughter and mother sides (Ortiz and Novick 2006). Although Chs $3 p$ is vital for septation, absence of the catalysing unit may be compensated by other chitin synthetases 
and the chs3 3 mutant produces a modified threelayered septum (Shaw et al. 1991). The chitin synthetase 3 impairment was related to the reduced volume of the final cell without changes in wall thickness. The AFM measurements reported by Dague et al. (2010) showed an increase in the wall surface roughness and a reduction in Young's modulus determined by indentation with a nano probe (Dague et al. 2010).

The chitin accumulation through the lifespan mostly as the ring structure at the budding neck was proposed as a possible constraint of longevity in yeast due to decrease in the available wall space for subsequent cell divisions (Mortimer and Johnston 1959), however, this suggestion has not been supported with experimental studies (Powell et al. 2003). The number of chitin bud scars indeed increases in the course of subsequent cell cycles (Egilmez et al. 1990) and is therefore considered a biomarker for replicative cell age (Powell et al. 2003). The scar tissue is flexible and may undergo some stretching as a result of cell expansion (Powell et al. 2003). However, this does not result in local discontinuity since the inelastic chitin ring undergoes breakage that is symmetrically distributed (Powell et al. 2003). The local damage is also prevented by tight founding of the chitin with $\beta-1,3-$ glucan in the cell wall (Arroyo and Arroyo 2013).

The cts $1 \Delta$ mutant's budding lifespan was much lower compared to chs3 4 and both strains were characterised by reduction in the final cell volume and some insignificant changes in wall thickness. Cts1 chitinase is one of essential enzymes, apart from a number of glucanases/glucanosyl transferases, needed for septa material degradation to enable mother and daughter cell separation. Since the septa is digested exclusively from the daughter's side, the mother cell remains after the cells dissociation with a remnant bud scar. This process of septa destruction is highly controlled to maintain integrity of the separated daughter cell, and therefore deletion of CTS1 may considerably affect the process of proliferation. Both chitin synthetase (chs3A) and chitinase (cts1A) mutants showed changes in the birth scar morphology as revealed in the SEM micrographs. These observations support earlier reports indicating their specific activity in the region of septum formation and motherdaughter cell separation.

The literature data (Dague et al. 2010) indicate a considerable decrease in wall stiffness and increase in wall surface roughness both at the exponential and stationary stages.

Cts1p plays a particularly important role in the digestion/degradation of the primary septum that is required for separation of mother and daughter cells. The CTS1 gene deletion may substantially affect this process. Although both enzymes are essential for formation of the septa, where Chs 3 contributes mainly to structural reinforcing, some compensatory mechanisms may be activated if chitin is not produced at this location (Schmidt 2004). However, it provides a rather minor direct contribution to the overall chitin synthesis and chitin content level in normal conditions. It is mostly implicated in the formation of the secondary septum specifically required for mother-daughter cell separation during the budding process. This interaction seems to explain the increase in wall surface roughness and reduction in Young's modulus determined by indentation with the atomic force microscopy probe (Dague et al. 2010). We could see that the mutations affecting the mother-daughter cell separation reduced the mother cell's longevity. Both mutants showed changes in the birth scar morphology as revealed in this study by SEM micrographs. In addition, these mutations reduced the final volume of mother cells but did not alter the cell wall thickness markedly.

The most dramatic reduction in the budding lifespan was observed for the mnn9 4 mutant defective in the synthesis of the $\alpha-1,6-$ mannan. The Mnn9 protein is a subunit of the mannosyltransferase complex located in Golgi membranes which takes part in the synthesis of the mannan backbone $N$-linked to the cell wall proteins (Yip et al. 1994). Glycosylated proteins are the main compound of the outer wall layer in fungi and the extent and pattern of protein glycosylation is essential for such cell functions as morphogenesis, wall adhesive properties and wall permeability (Orlean 2012). Due to a relatively high content of the compound in cell wall any disturbance in its biosynthesis must affect the wall's integrity. Additionally, this mutation may affect directly the budding longevity through generation of abnormalities in the septum, which in the case of mnn94 is much thinner or shows signs of repair or remediation (Schmidt et al. 2005). The cell wall thickness and the rigidity of the mutant were unchanged, probably because the defect in the mannosylation was compensated by a many-fold increase in the chitin content and 
a slight increase in the amount of glucans (Dague et al. 2010). The mutation inhibited cell volumetric growth at budding; however, it dramatically increased wall permeability and wall surface roughness.

Extended cell longevity was observed exclusively for the knr4 $\Delta$ mutant. The Knr4 protein is a transcriptional regulator of cell wall chitin and glucan synthesis (Hong et al. 1994). In this way Knr4p is involved in coordinating cell cycle advancement with cell wall integrity. The mutant showed a slight increase in cell wall rigidity as revealed by AFM probe indentation and a marked increase in wall surface roughness in the exponential growth stage. The latter could be the result of an increase in the mannan content (Dague et al. 2010). The improvement in the mechanical properties, despite decrease in the $\beta$-glucan content, might be explained by the marked increase in chitin content (Hong et al. 1994) that could contribute to establishing more abundant wall polymer interlinking.

As asexual reproduction, budding is unavoidably associated with a continuous growth of the mother cell's volume (Molon and Zadrag-Tecza 2016a; Zadrag-Tecza et al. 2009). Therefore, we subsequently attempted to address the changes in budding lifespan referring to the volume which the cell may achieve in the case of a given wall mutation, and thus verify the hypertrophy hypothesis that has been hotly debated recently (Bilinski and Bartosz 2006; Ganley et al. 2012; Kaeberlein 2012; Wright et al. 2013; Yang et al. 2011). This hypothesis assumes that cell volume is a major factor determining finished budding (Bilinski and Bartosz 2006; Bilinski et al. 2012). Further papers suggest that the rate of volume growth is a factor regulating the parameter (Yang et al. 2011). In support of the hypertrophy hypothesis, studies on various mutations (Yang et al. 2011) and nutrition conditions (Turner et al. 2012) were reported. There is also some evidence against the argument. Moretto et al. (2013) showed that the knock-out FOBI gene increases budding lifespan without altering cell volume (Moretto et al. 2013). Additionally, diploid yeast has larger cells than haploid, and shows an increased lifespan compared to the haploid cells (Kaeberlein et al. 2005). Latest data suggest that yeast cells can achieve a significantly higher volume than the wild-type strain (Molon and Zadrag-Tecza 2016a). In turn, while exploring the cell wall mutants, we show here that yeast cells gain a 50\% smaller maximum volume than the wild-type strain. What is interesting, in the case of the cts $1 \Delta$, chs34 and mnn94 mutants the cells achieved various budding lifespans for the same cell volumes. In our other studies (Molon and ZadragTecza 2016a), cells of some mutants (sfpl $\Delta$ ) showing decreased budding lifespan reached dramatically enhanced volumes compared to the wild-type strain. Therefore, cells may display a wide range of volume in the same genetic backgrounds and under the same environmental conditions when they stop budding. To sum up, our data and all of the aforementioned reports support the conviction that achieving hypertrophy cannot be the only factor that determines the budding lifespan of the cell. Impact of hypertrophy on aging requires therefore further studies and clarification.

Using multiple imaging techniques, e.g. SEM, TEM and AFM, and the spoil test, as well as taking into consideration indentation measurements of wall stiffness reported in literature (Dague et al. 2010), we attempted to establish a putative association between the phenotypic modifications in the cell wall and the changes in the budding lifespan. However, we did not find any clear association between cell wall characteristics or cell morphological traits and the budding lifespan, even though the disturbances in wall biosynthesis had a pronounced effect on both the lifespan and morphology of cells. This may be a consequence of extended molecular and biochemical adaptive responses of cells to the point mutations in cell wall biosynthesis. The response could be driven by sensing of the wall mechanical status and activation of the cell wall integrity (CWI) pathway (Levin 2011), which affects multiple cellular processes. It may, among other things, induce a complex compensatory alteration of cell wall composition, e.g. hyperaccumulation of chitin (Popolo et al. 1997; Ram et al. 1998), which may have impact on the septation process and the mother-daughter cell separation. In addition, more recent studies have indicated that the mitogen-activated protein kinase (MAPK)-mediated signalling pathway activated by cell wall stressors affects the cell cycle progression (Carbo and Perez-Martin 2010) which may influence the budding lifespan.

The above may suggest, therefore, that establishing a cross talk between the CWI pathway and the signalling networks controlling the aging process might provide a better understanding of the complex mechanism of the budding lifespan. 
Acknowledgement This research was supported by the University of Rzeszow task grant No. WBR/KBiBK/DS/1/2016.

\section{Compliance with ethical standards}

Conflict of interest The authors declare that they have no conflict of interest.

Open Access This article is distributed under the terms of the Creative Commons Attribution 4.0 International License (http:// creativecommons.org/licenses/by/4.0/), which permits unrestricted use, distribution, and reproduction in any medium, provided you give appropriate credit to the original author(s) and the source, provide a link to the Creative Commons license, and indicate if changes were made.

\section{References}

Aguilaniu H, Gustafsson L, Rigoulet M, Nystrom T (2003) Asymmetric inheritance of oxidatively damaged proteins during cytokinesis. Science 299:1751-1753

Arroyo ECJ, Arroyo J (2013) How carbohydrates sculpt cells: chemical control of morphogenesis in the yeast cell wall. Nat Rev Microbiol 11:648-655

Bartholomew JW, Mittwer T (1953) Demonstration of yeast bud scars with the electron microscope. J Bacteriol 65:272-275

Barton AA (1950) Some aspects of cell division in Saccharomyces cerevisiae. J Gen Microbiol 4:84-86

Bilinski T, Bartosz G (2006) Hypothesis: cell volume limits cell divisions. Acta Biochim Pol 53:833-835

Bilinski T, Zadrag-Tecza R, Bartosz G (2012) Hypertrophy hypothesis as an alternative explanation of the phenomenon of replicative aging of yeast. FEMS Yeast Res 12:97-101

Bulik DA, Olczak M, Lucero HA, Osmond BC, Robbins PW, Specht CA (2003) Chitin synthesis in Saccharomyces cerevisiae in response to supplementation of growth medium with glucosamine and cell wall stress. Eukaryot Cell 2:886-900

Cabib E, Schmidt M (2003) Chitin synthase III activity, but not the chitin ring, is required for remedial septa formation in budding yeast. FEMS Microbiol Lett 224:299-305

Cabib E, Drgon T, Drgonova J, Ford RA, Kollar R (1997) The yeast cell wall, a dynamic structure engaged in growth and morphogenesis. Biochem Soc Trans 25:200-204

Cabib E, Roh DH, Schmidt M, Crotti LB, Varma A (2001) The yeast cell wall and septum as paradigms of cell growth and morphogenesis. J Biol Chem 276:19679-19682

Carbo N, Perez-Martin J (2010) Activation of the cell wall integrity pathway promotes escape from $\mathrm{G} 2$ in the fungus Ustilago maydis. PLoS Genet. https://doi.org/10.1371/ journal.pgen.1001009

Carotti C, Ferrario L, Roncero C, Valdivieso MH, Duran A, Popolo L (2002) Maintenance of cell integrity in the gas1 mutant of Saccharomyces cerevisiae requires the Chs3ptargeting and activation pathway and involves an unusual Chs3p localization. Yeast 19:1113-1124

Dague E, Bitar R, Ranchon H, Durand F, Yken HM, Francois JM (2010) An atomic force microscopy analysis of yeast mutants defective in cell wall architecture. Yeast 27:673-684

de Groot PWJ et al (2001) A genomic approach for the identification and classification of genes involved in cell wall formation and its regulation in Saccharomyces cerevisiae. Comp Funct Genomics 2:124-142

Egilmez NK, Jazwinski SM (1989) Evidence for the involvement of a cytoplasmic factor in the aging of the yeast Saccharomyces cerevisiae. J Bacteriol 171:37-42

Egilmez NK, Chen JB, Jazwinski SM (1990) Preparation and partial characterization of old yeast-cells. J Gerontol 45:B9-B17

Erjavec N, Larsson L, Grantham J, Nystrom T (2007) Accelerated aging and failure to segregate damaged proteins in Sir2 mutants can be suppressed by overproducing the protein aggregation-remodeling factor Hsp104p. Genes Dev 21:2410-2421

Ganley ARD, Breitenbach M, Kennedy BK, Kobayashi T et al (2012) Yeast hypertrophy: cause or consequence of aging? Reply to Bilinski. FEMS Yeast Res 12:267-268

Gow NAR, Latge JP, Munro CA (2017) The fungal cell wall: structure, biosynthesis, and function. Microbiol Spectr. https://doi.org/10.1128/microbiolspec.FUNK-0035-2016

Hong Z, Mann P, Brown NH, Tran LE, Shaw KJ, Hare RS, Didomenico B (1994) Cloning and characterization of $k n r 4$, a yeast gene involved in (1,3)-beta-glucan synthesis. Mol Cell Biol 14:1017-1025

Kaeberlein M, Kirkland KT, Fields S, Kennedy BK (2005) Genes determining yeast replicative life span in a longlived genetic background. Mech Ageing Dev 126:491-504

Kaeberlein M et al (2012) Hypertrophy and senescence factors in yeast aging. A reply to Bilinski. FEMS Yeast Res 12:269-270

Klis FM, Mol P, Hellingwerf K, Brul S (2002) Dynamics of cell wall structure in Saccharomyces cerevisiae. FEMS Microbiol Rev 26(3):239-256

Klis FM, Boorsma A, De Groot PWJ (2006) Cell wall construction in Saccharomyces cerevisiae. Yeast 23:185-202

Kuranda MJ, Robbins PW (1991) Chitinase is required for cellseparation during growth of Saccharomyces cerevisiae. J Biol Chem 266:19758-19767

Lesage G, Bussey H (2006) Cell wall assembly in Saccharomyces cerevisiae. Microbiol Mol Biol Rev 70:317-343

Lesage G, Shapiro J, Specht CA, Sdicu AM, Menard P, Hussein S, Tong AHY, Boone C, Bussey H (2005) An interactional network of genes involved in chitin synthesis in Saccharomyces cerevisiae. BMC Genet. https://doi.org/10.1186/ 1471-2156-6-8

Levin DE (2011) Regulation of cell wall biogenesis in Saccharomyces cerevisiae: the cell wall integrity signaling pathway. Genetics 189:1145-1175

Lippuner AD, Julou T, Barral Y (2014) Budding yeast as a model organism to study the effects of age. FEMS Microbiol Rev 38:300-325

Molon M, Zadrag-Tecza R (2016a) Effect of temperature on replicative aging of the budding yeast Saccharomyces cerevisiae. Biogerontology 17:347-357

Molon M, Zadrag-Tecza R (2016b) The links between hypertrophy, reproductive potential and longevity in the Saccharomyces cerevisiae yeast. Acta Biochim Pol 63:329-334 
Molon M, Szajwaj M, Tchorzewski M, Skoczowski A, Niewiadomska E, Zadrag-Tecza R (2016) The rate of metabolism as a factor determining longevity of the Saccharomyces cerevisiae yeast. Age 38(1): 11

Moretto F, Sagot I, Daignan-Fornier B, Pinson B (2013) A pharmaco-epistasis strategy reveals a new cell size controlling pathway in yeast. Mol Syst Biol 9:707

Mortimer RK, Johnston JR (1959) Life span of individual yeast cells. Nature 183:1751-1752

Orlean P (2012) Architecture and biosynthesis of the Saccharomyces cerevisiae cell wall. Genetics 192:775-818

Ortiz D, Novick PJ (2006) Ypt32p regulates the translocation of Chs $3 p$ from an internal pool to the plasma membrane. Eur J Cell Biol 85:107-116

Popolo L, Gilardelli D, Bonfante P, Vai M (1997) Increase in chitin as an essential response to defects in assembly of cell wall polymers in the ggp1 Delta mutant of Saccharomyces cerevisiae. J Bacteriol 179:463-469

Powell CD, Van Zandycke SM, Quain DE, Smart KA (2000) Replicative ageing and senescence in Saccharomyces cerevisiae and the impact on brewing fermentations. Microbiology-Sgm 146:1023-1034

Powell CD, Quain DE, Smart KA (2003) Chitin scar breaks in aged Saccharomyces cerevisiae. Microbiology-Sgm 149:3129-3137

Ram AFJ, Kapteyn JC, Montijn RC, Caro LHP, Douwes JE, Baginsky W, Mazur P, Van den Ende H, Klis FM (1998) Loss of the plasma membrane-bound protein Gas1p in Saccharomyces cerevisiae results in the release of beta 1,3glucan into the medium and induces a compensation mechanism to ensure cell wall integrity. J Bacteriol 180:1418-1424

Roncero C, Sanchez Y (2010) Cell separation and the maintenance of cell integrity during cytokinesis in yeast: the assembly of a septum. Yeast 27:521-530

Schmidt M (2004) Survival and cytokinesis of Saccharomyces cerevisiae in the absence of chitin. Microbiology-Sgm 150:3253-3260

Schmidt M, Bowers B, Varma A, Roh DH, Cabib E (2002) In budding yeast, contraction of the actomyosin ring and formation of the primary septum at cytokinesis depend on each other. J Cell Sci 115:293-302

Schmidt M, Strenk ME, Boyer MP, Fritsch BJ (2005) Importance of cell wall mannoproteins for septum formation in Saccharomyces cerevisiae. Yeast 22:715-723

Shaw JA, Mol PC, Bowers B, Silverman SJ, Valdivieso MH, Duran A, Cabib E (1991) The function of chitin synthase-2 and synthase-3 in the Saccharomyces cerevisiae cell-cycle. J Cell Biol 114:111-123

Sinclair DA, Guarente L (1997) Extrachromosomal rDNA circles-a cause of aging in yeast. Cell 91:1033-1042

Sinclair DA, Mills K, Guarente L (1997) Accelerated aging and nucleolar fragmentation yeast sgs1 mutants. Science 277:1313-1316

Smith AE, Zhang ZB, Thomas CR, Moxham KE, Middelberg APJ (2000) The mechanical properties of Saccharomyces cerevisiae. Proc Natl Acad Sci USA 97:9871-9874

Steinkraus KA, Kaeberlein M, Kennedy BK (2008) Replicative aging in yeast: the means to the end. Annu Rev Cell Dev Biol 24:29-54

Tolliday N, Pitcher M, Li R (2003) Direct evidence for a critical role of myosin II in budding yeast cytokinesis and the evolvability of new cytokinetic mechanisms in the absence of myosin II. Mol Biol Cell 14:798-809

Turner JJ, Ewald JC, Skotheim JM (2012) Cell size control in yeast. Curr Biol 22:R350-R359

Utsugi T, Minemura M, Hirata A, Abe M, Watanabe D, Ohya Y (2002) Movement of yeast 1,3-beta-glucan synthase is essential for uniform cell wall synthesis. Genes Cells 7:1-9

Wawryn J, Krzepilko A, Myszka A, Bilinski T (1999) Deficiency in superoxide dismutases shortens life span of yeast cells. Acta Biochim Pol 46:249-253

Wright J, Dungrawala H, Bright RK, Schneider BL (2013) A growing role for hypertrophy in senescence. FEMS Yeast Res 13:2-6

Yang JY, Dungrawala H, Hua H, Manukyan A, Abraham L, Lane W, Mead H, Wright J, Schneider BL (2011) Cell size and growth rate are major determinants of replicative lifespan. Cell Cycle 10:144-155

Yip CL, Welch SK, Klebl F, Gilbert T, Seidel P, Grant FJ, Ohara PJ, Mackay VL (1994) Cloning and analysis of the Saccharomyces cerevisiae MNN9 AND MNN1 genes required for complex glycosylation of secreted proteins. Proc Natl Acad Sci USA 91:2723-2727

Zadrag-Tecza R, Kwolek-Mirek M, Bartosz G, Bilinski T (2009) Cell volume as a factor limiting the replicative lifespan of the yeast Saccharomyces cerevisiae. Biogerontology 10:481-488

Ziman M, Chuang JS, Tsung M, Hamamoto S, Schekman R (1998) Chs6p-dependent anterograde transport of Chs3p from the chitosome to the plasma membrane in Saccharomyces cerevisiae. Mol Biol Cell 9:1565-1576 